\title{
Radiation pattern control of microstrip antenna in elevation and azimuth planes using EBG and pin diode
}

\author{
M.K.Abdulhameed ${ }^{1}$, M.S.Mohamad Isa ${ }^{2}$, Z.Zakaria ${ }^{3}$, I.M. Ibrahim ${ }^{4}$, Mowafak K.Mohsen ${ }^{5}$ \\ 1,2,3,4,5 Centre for Telecommunication Research and Innovation (CeTRI), Faculty of Electronic and Computer Engineering, \\ Universiti Teknikal Malaysia Melaka (UTeM), Malaysia \\ ${ }^{1,5}$ Ministry of Higher Education and Scientific Research, University of Kerbala, Iraq
}

\begin{tabular}{l} 
Article Info \\
\hline Article history: \\
Received Jul 3, 2018 \\
Revised Jul 24, 2018 \\
Accepted Jul 29, 2018 \\
\hline
\end{tabular}

\section{Keywords:}

$\mathrm{E}$ and $\mathrm{Z}$ planes radiation pattern control

Electromagnetic Band-Gap

Microstrip Antenna

Mushroom-like EBG structure

Switchable EBG

\begin{abstract}
An important issue in wireless communication systems, which is related to the antenna gain degradation in case of changing the main direction of the antenna radiation pattern, this variation is not approval in many communications systems. In order to improve antenna radiation performances, Electromagnetic band gap (EBG)-antenna with radiation pattern control capability is presented. Mushroom-like EBG structure for suppressing surface waves has been combined, with the switching diode to produce the radiation pattern control with improving antenna characteristics of gain, directivity and efficiency. EBG of several cells are surrounded the patch antenna and placed symmetrically for the two opposite sides, generating different radiation patterns control ability in both the elevation (E) $\left(-20^{\circ}<\varphi<20^{\circ}\right)$ and azimuth (Z) planes $\left(-18^{\circ}<\theta<18^{\circ}\right)$. At the ground plane of antenna the diodes have been switched ON and OFF states, the EBG sector properties in stop band (connecting vias) and pass band (disconnecting vias) are altered. Using CST Microwave Studio (CST MWS) the results show the flexibility in radiation pattern control for the $\mathrm{Z}$ and $\mathrm{E}$ planes using only four diodes. Antenna directivity of $10 \mathrm{dBi}$, gain $9.86 \mathrm{~dB}$ and efficiency $96.5 \%$ at the operating frequency of $6 \mathrm{GHz}$, more results for all direction has been stated in Table1. Significantly, unlike a conventional beam steering, this method does not suffering from gain degradation and the main lobe gain is approximately constant for all steering angles.
\end{abstract}

Copyright (C) 2019 Institute of Advanced Engineering and Science. All rights reserved.

\section{Corresponding Author:}

M.K.Abdulhameed,

Department of Telecommunication Engineering,

Faculty of Electronic and Computer Engineering,

Universiti Teknikal Malaysia Melaka,

Hang Tuah Jaya, 76100 Durian Tunggal, Melaka, Malaysia.

Email: eng_mka@yahoo.com

\section{INTRODUCTION}

Microstrip patch antennas have been applied commonly in numerous purposes due to low profile, low cost, light-weight and appropriate of integration with RF devices [1], [2]. The EBG periodic structure has been applied to microwave planar waveguides can result in pass or stop bands. By accurate choice of dimensions and periods specific waves are permitted for propagatting, whereas the other waves like surface waves can be suppressed. Surface waves decrease the efficiency of antenna and gain, reduce bandwidth, rise the radiation of end-fire and cross-polarization levels. The mushroom-like EBG surface has been described in [3] and the other explanation in [4] of the uni-planar EBG surface has been used to surround a microstrip patch antenna for reducing the surface wave effect. The feature of surface-waves suppression supports to reducing backward direction and the amount of power wasted [5], leads to improve antenna performance [6]-[14] by raising the gain of antenna [15] and reduction in antenna size [16]. The antennas 
with beam steering ability have a lot of advantage by getting better range in channel capacity while lessening the interference [17]. When considering on inserting additional features to current antennas, the main question is the applications and advantages of doing so. When the single antenna can be used in multiple systems, this called reconfigured antenna and it will be an important feature in wireless communication systems. Integrated the antenna with Radio Frequency (RF) switches, antenna structure can be altered (reconfigurable antenna) [18]. Four types of the reconfigurable antenna can be achieved. Classified as reconfiguration process in radiation pattern, operation frequency, polarization behavior, or a combination between any of these types [19].

The main function of the reconfigurable radiation pattern antenna, in controlling the radiation pattern into many directions. One of the main problems related to the microstrip antenna is the surface waves excitations, especially when changing the direction for the main lobe of the radiation pattern, which caused the degredation in the antenna gain. With advanced fabrication techniques for antenna, beam steering in lower complex and miniature patch antennas can be achieved. Several techniques can be applied to realize the radiation pattern control along the Z-plane (azimuth plane). Used of electromagnetic bandgap (EBG) structure was one of these mechanismss [20]-[22]. Meta-material and electromagnetic surface design and development have contributed towards the evolvement of surface wave antenna which had effectively reduced the overall antenna size with total improvements in the overall performance. Various designs and shapes of EBG structures have been implemented on the metal surfaces of dielectric substrates in order to stop and propagate the surface wave [23]. Artificial periodic structures of EBG demonstrated the band stop features since no electromagnetic wave propagation is permitted. Therefore, radiation pattern control can be achieved by using switching devices to control the transmission characteristics of the EBG structure.

In this paper, a low profile antenna for radiation pattern control in both Azimuth and Elevation planes is designed. Rectangular patch antenna has been surrounded by square cells of EBG structure for suppressing the surface waves on the high dielectric constant substrate. A band gap of the EBG can be generated for frequencies at the same band of the operating frequency, by placing the (EBGs) periodically on the antenna substrate. Antenna structure that results from this design can prevent the excited surface waves along the substrate from the propagation. Radiation power can be propagated to a space wave better than wasted as surface waves in the substrate. The EBG structure with switched stop band and pass band features around the $6 \mathrm{GHz}$ band gap has been designed. A beam steering antenna using the switched diode with EBG surface is presented, by connecting and disconnecting the pin vias to the ground plane for all of the antenna sides we achieved radiation control in $\mathrm{E}$ and $\mathrm{Z}$ planes. The diodes at the ground plane have been used in $\mathrm{ON}$ state for connecting all the Pin vias of EBG elements to the ground plane, or in OFF state for disconnecting all of these pin vias from the ground plane of antenna to steer the beam into desired sector. Unconventional radiation pattern control is a unique characteristic of this design; the main lobe gain is approximately constant for all steering angles in E-plane with unnoticeable degradation of gain in Z-plane direction.

\section{EBG STRUCTURE AND DESIGN}

The mushroom-like EBG structure, which is actually a 2-D EBG surface, was initially proposed by [3]. There were four parts in the proposed model: i) ground plane, ii) dielectric substrate, iii) metallic patches, and iv) connecting vias. A distinct feature of stopband was exhibited by these EBG structures for surface-wave propagation. An LC filter array can be used for the explanation of the operation mechanism of the EBG structure, as shown in Figure 1c. The current, which is flowing through vias, causes the inductor $L$ effect, whereas the gap among the neighboring patches resulted in the capacitor $C$ effect. As shown in Figure 1a. $\mathrm{W}$ is the patch width, gap width is $g$, thickness of substrate is $h$ and $\epsilon_{\mathrm{r}}$ is dielectric constant.

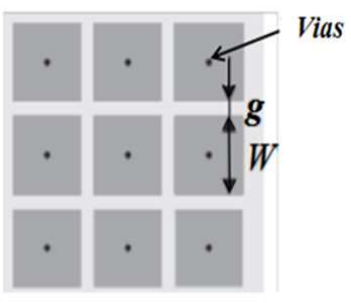

(a)

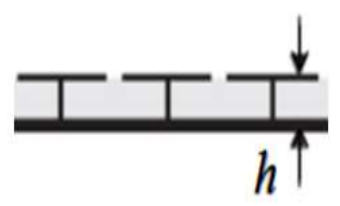

(b)

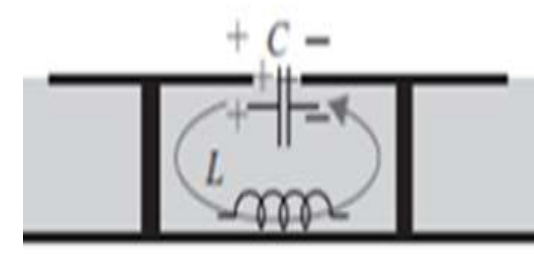

(c)

Figure 1. Geometries of mushroom like EBG (a) Top view (b) Cross view (c) Lumped LC typical analysis [3] 
The EBG structure operation can be designed by an LC filter equivalent circuit model if periodicity of structure is as small as possible in comparison with the wavelength of operating frequency [24]. The impedance of the structure is very high at resonant frequency, and hence the structure avoids the emission of any surface waves. This results in a band gap of frequency [3]. A band gap of the EBG can be generated for frequencies at the same band of the operating frequency, by placing the (EBGs) periodically on the antenna substrate. Antenna structure that results from this design can prevent the excited surface waves along the substrate from the propagation. The second model is the helpful band gap for suppressing surface wave and the frequency region where the field of structure is in case of phase reflection and the reflection coefficient properties are extracted analytically in [25],[26].

The transmission line model for plane waves additional to surface waves will explain at this section, to cover the frequency of $6 \mathrm{GHz}$, CST software has been used to tune the values of $\mathrm{W}$ and $\mathrm{g}$. The optimization of the EBG dimensions has been implemented using the microstrip transmission line technique which measuring the surface mutual coupling within two edges as shown in Figure 2(a). The width of the transmission line is determined as $1.5 \mathrm{~mm}$, which has been calculated using CST MWS to achieve matching with the $50 \Omega$ input impedance. A couple of $50 \Omega$ discrete ports have been connected at both edges of the transmission line for calculating the EBG surface mutual coupling. The EBG surface mutual coupling, as shown on Figure 3, represents the band gap characteristics for the EBG with optimized parameters that covers the antenna operating frequency of $6 \mathrm{GHz}$. Where EBG width $W=3 \mathrm{~mm}\left(0.06 \lambda_{\mathrm{o}}\right), g=0.3 \mathrm{~mm}\left(0.006 \lambda_{\mathrm{o}}\right)$ and via radius of $0.5 \mathrm{~mm}\left(0.01 \lambda_{\mathrm{o}}\right)$ connected between the ground plane and patch on a Rogers RT/Duroid 6010 substrate of height $h=2 \mathrm{~mm}\left(0.04 \lambda_{\mathrm{o}}\right)$, relative permittivity of 10.2 . The mutual coupling for the EBG structure illustrates the enhancement in EBG surface mutual coupling when the pin vias are removed. Additionally the results when the pin vias are inserted illustrate that the EBG structure rejects the surface wave propagation. Hence, the structure characteristics can be easily altered by alternately switching the pin vias IN and OUT. The main idea of this research is to drops the resonant frequency of antenna within the EBG band gap and then the surface waves could be forbidden. The EBG structure has been designed to achieve a stop band about the band of $6 \mathrm{GHz}$, in case of connecting the pin vias to the antenna ground plane, and a pass band at the same band of frequency when the pin vias have been disconnected from the ground plane of antenna. As shown in Figure 2(b) the simulation for the transmission characteristics of the suggested EBG structure when the pin vias are inserted and removed.

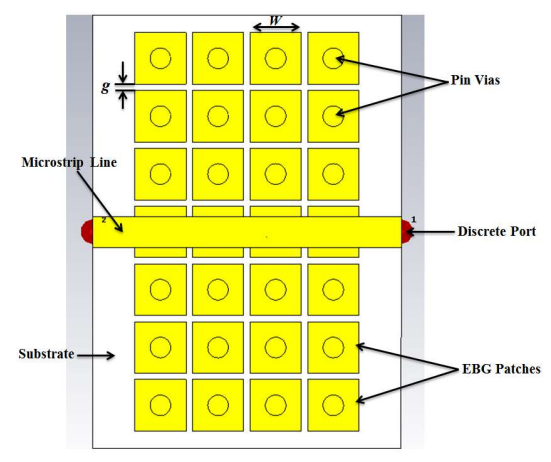

(a)

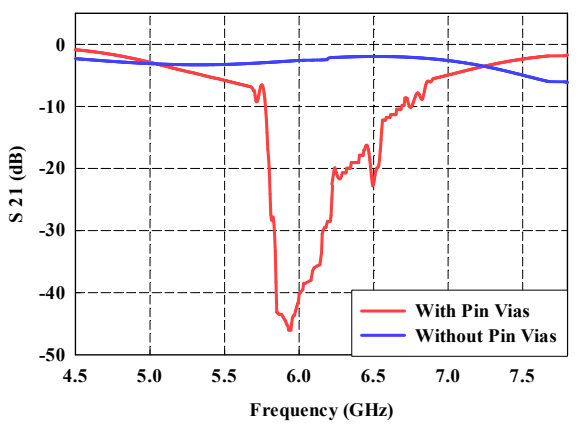

(b)

Figure 2. (a) EBG microstrip transmission line and

(b) Transmission characteristics of EBG structure

\section{ANTENNA DESIGN WITH EBG AND PIN DIODE}

Patch antennas in various sizes and shapes are available. Rectangular patch is a most simple and easy to fabricate. The size of the antenna reduces as the relative dielectric constant of the substrate increase [27]. In this research, we designed a rectangular patch antenna with probe feed on the Rogers RT/Duroid 6010, dielectric constant $\epsilon_{\mathrm{r}}=10.2$ and the substrate thickness is $h=2 \mathrm{~mm}\left(0.04 \lambda_{\mathrm{o}}\right)$. The lengths of the patch are $6.1 \mathrm{~mm}\left(0.124 \lambda_{\mathrm{o}}\right)$ by $7.4 \mathrm{~mm}\left(0.148 \lambda_{\mathrm{o}}\right)$. By surrounding the rectangular patch antenna with 4 columns, each of the upper and downer sides are composed of fifty four square-patches mushroom EBG unit cell and each of the left and right sides are composed of twenty eight square-patches mushroom EBG unit cell or four columns of EBGs shifted inwards to antenna edges by $0.3 \mathrm{~mm}\left(0.06 \lambda_{\mathrm{o}}\right)$ equal to gap of its 
design. Structures of EBG were sited more than two periods away from the patch antenna edges. Unlike uniplanar and other EBGs, in mushroom-like structures there are no interconnecting microstrip lines that limit their arrangement cyclically. Using this feature, the structures of EBG have been shifted inwards to the antenna ends as shown in Figure 3(a). A switching pin diode is used for connecting the centers of the EBG square patches to the ground plane as shown in Figure 3(b), where we make a slot of $0.3 \mathrm{~mm}$ on the ground plane of antenna which surrounded all of the EBG patches with pin vias in the four sides of antenna. In case of diode in $\mathrm{ON}$ state the slot connected to ground plane and by doing this the pin vias will connect to the antenna ground plane. Stop band of frequency when the pin vias have been connected. Frequency band pass when the pin vias have been disconnected from the ground plane of antenna where the diode in OFF state. The slots in the ground plane for each sector helps to reduce the diodes number to four, one in each sector and thus, lowering the influence of the biasing circuit on the features of antenna. The antenna pattern directivity has been controlled by connecting and disconnecting pin vias in each sector to vary the beam direction. Simulation results were presented in the next section.

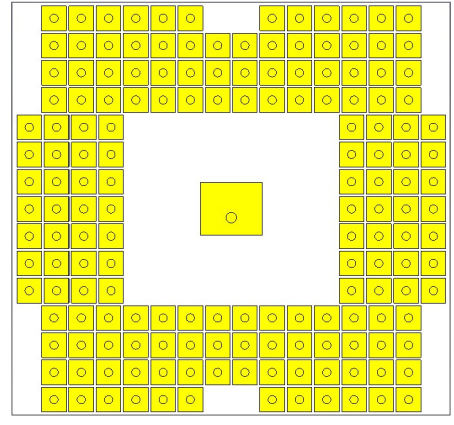

(a)

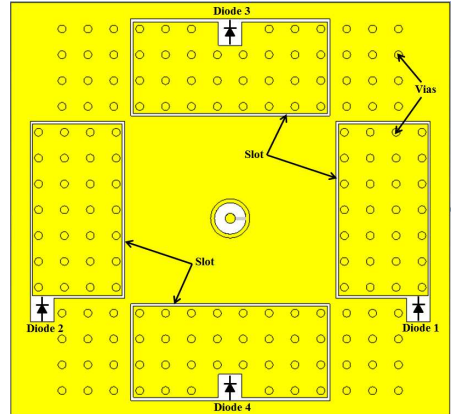

(b)

Figure 3. (a) A rectangular patch antenna surrounded by mushroom-like EBG structures, (b) Back view (ground plane) of a rectangular patch antenna, four slots and pin diodes

\section{RESULT AND ANALYSIS}

In the previous section, the design of the rectangular patch antenna in $6 \mathrm{GHz}$ and the EBG with the Band gap for covering $6 \mathrm{GHz}$ are presented. Simulations were done for the surrounding antenna by EBG and the referance antenna in order to compare the performance. As shown in Figure 3(b) all of the four diodes are setting as ON states (short circuit), for showing the suppressing surface waves and its effects on antenna improvement in terms of gain, directivity and efficiency of antenna. It is observed that when using four columns at left and right sides with shifting inwards to the antenna ends by $0.3 \mathrm{~mm}\left(0.06 \lambda_{\mathrm{o}}\right)$ as shown in Figure 3(a). The simulated S-parameters in terms of S-11 was established that the good matching S-11 at $6 \mathrm{GHz}$. The configurations shifted the resonant frequency of the antenna slightly to $5.99 \mathrm{GHz}$ when using EBG with good matching S-11 of less than $-30 \mathrm{~dB}$, while the resonance frequency for the antenna without using EBG pointed at $6.01 \mathrm{GHz}$ with matching S-11 of less than $-20 \mathrm{~dB}$ as shown in Figure 4(a).

The stop band for suppressing surface wave will appear for all antenna borders and no more waves will propagate at these four direction. The rectangular patch antenna without EBG has $-7 \mathrm{~dB}$ side lobes, this side lobe will decrease the gain of antenna. Additionally; the main lobe has a beam width of about $87.3^{\circ}$. This big aperture angle will decrease the antenna directivity. The electric field radiation properties of the antenna with EBG observed the better reduction in side lobe level around $-17.5 \mathrm{~dB}$. Further, the beam width is about $57^{\circ}$ due to the suppressing surface waves at stop band of EBG. These features will generate a better gain and directivity in the direction of broadside of the antenna in comparison to the rectangular patch antenna without EBG. The measured directivity of a rectangular patch antenna with EBG elements is $10 \mathrm{dBi}$ and the gain is $9.86 \mathrm{~dB}$, at $0^{\circ}$ main lobe direction while the directivity and gain for the rectangular antenna without using EBG are $5.66 \mathrm{dBi}$ and $4.74 \mathrm{~dB}$ as shown in Figure 4(b) and Figure 4(c) respectively. 


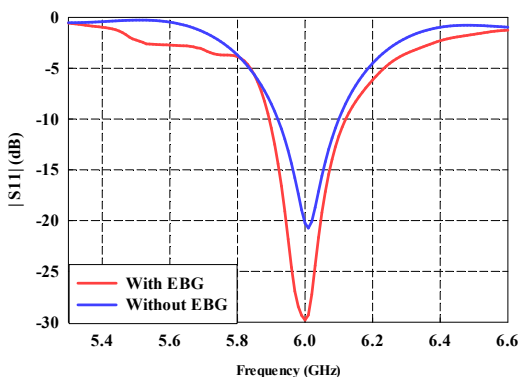

(a)

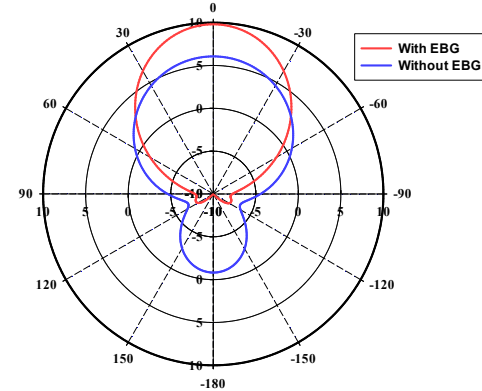

(b)

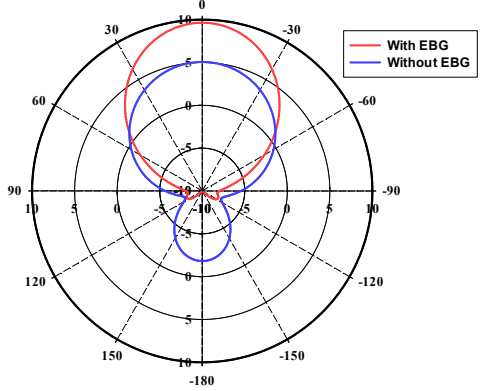

(c)

Figure 4. (a) Return loss of the microstrip antennas with and without EBG, (b) Directivity with and without EBG at $0^{\circ}$ main lobe direction (c) Gain with and without EBG at $0^{\circ}$ main lobe direction

\subsection{Radiation pattern control on the elevation $(E)$ plane}

For controlling radiation pattern in the E-plane, both of diode 3 and diode4 has been switched ON. The switching will be either in diode 1 or diode 2, First case when the diode 1 was switched OFF and at the same time all of the rest diodes have been switched ON as shown in Figure 5(a), the stop band will appear on the all of antenna sides which has ON state of diodes and the pass band will present at the right side of antenna where diode1 has been switched OFF. The simulated power pattern of the antenna with EBG is directed at the elevation plane (E-plane) of the microstrip patch antenna from the left side of antenna (stop band ) where the diode2 is ON state (connecting Vias) to the right side of antenna (pass band) where the diode1 is OFF (disconnecting Vias) in the direction of $20^{\circ}$ as shown in Figure 5(b).

The mushroom-like EBG has been simulated to explain its capability to control the power pattern directivity around the elevation plane (E-plane) of the microstrip patch antenna. By alternately switching diode the EBG sector surface characteristic will change also. Pin vias have been connected and disconnected at each sector to control the beam direction. For this case, to direct the pattern towards the right sector, pin vias at the sector have been disconnected from the ground plane (diodel switched OFF), whereas pin vias have been connected to ground plane of antenna for the left sector through switched the diode 2 at ON state. As a result, the surface wave has propagated towards right sector and reflected from the left sector. Figure 5(c) shows the Theta-plane power pattern at phi $=0^{\circ}$, which is directed towards the right sector at theta $=+20^{\circ}$ with the maximum gain and directivity which equal to $9.4 \mathrm{~dB}$ and $9.7 \mathrm{dBi}$ respectively.

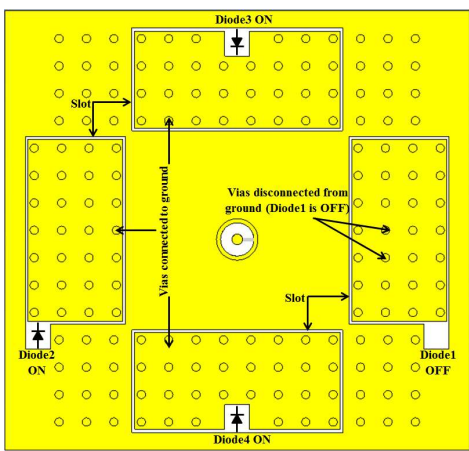

(a)

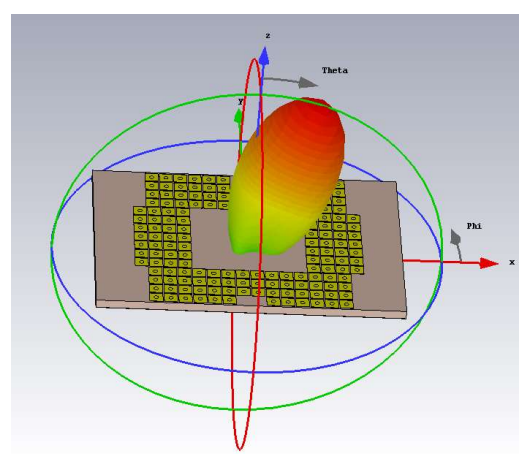

(b)

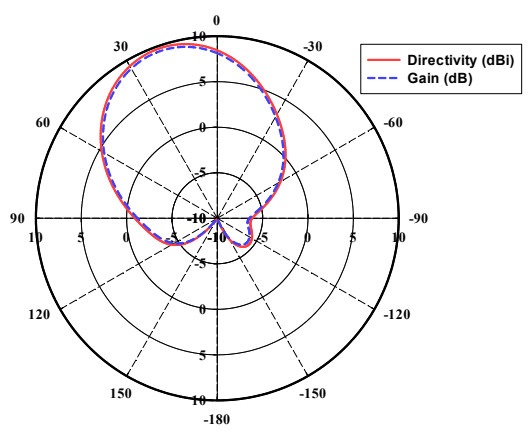

(c)

Figure 5. First Case of E-plane: (a) Diodes (2,3and4) are setting as ON state and Diode1 is OFF state, (b) Radiation Pattern is directed to the direction of $+20^{\circ}$ and (c) Power patterns in the E-plane at theta $=+20^{\circ}$

Second case when the diode1 on the right side of antenna has been switched ON (short circuit) and the diode2 on the left side was switched OFF (open circuit) as shown in Figure 6(a), the stop band will appear on the right side and the pass band will present at the left side of antenna. The simulated antenna radiation pattern with EBG is directed from the right side of antenna (stop band) where the diode1 is ON 
state (connecting vias) to the left side (pass band) where the diode2 is OFF state (disconnecting vias) in the direction of $-20^{\circ}$ as shown in Figure 6(b).

Same arrangement method of EBGs for the previous case to clarify its capability to control the radiation pattern directivity around the elevation plane (E-plane) of the microstrip patch antenna. by alternately switching diode the EBG sector surface characteristic will change also. To direct the pattern towards the left sector, pin vias at the sector have been disconnected from the ground plane of antenna (diode2 switched OFF), whereas pin vias have been connected to the antenna ground plane (diode1 switched $\mathrm{ON}$ ) for the right sector. As a result, the surface wave has propagated towards left sector (pass band) and reflected from the right sector(stop band). Figure 6(c) shows the Theta-plane power pattern at phi $=0^{\circ}$, which is directed towards the left sector at theta $=-20^{\circ}$ with the maximum gain and directivity which equal to previous case $9.4 \mathrm{~dB}$ and $9.7 \mathrm{dBi}$ respectively. This approach does not suffer from gain degradation and the main lobe gain and directivity are approximately constant for all steering angles.

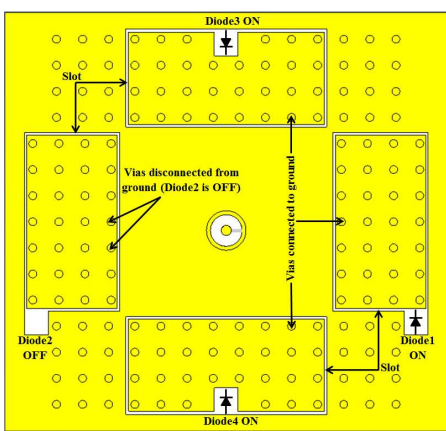

(a)

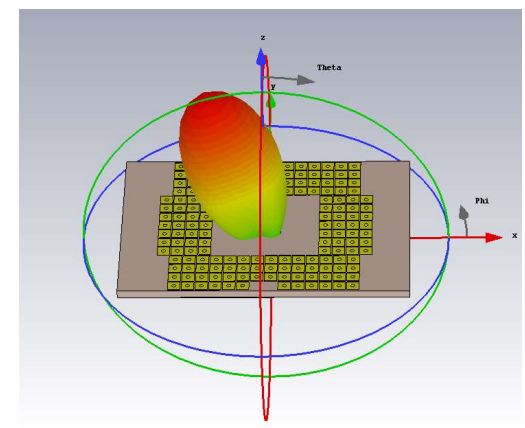

(b)

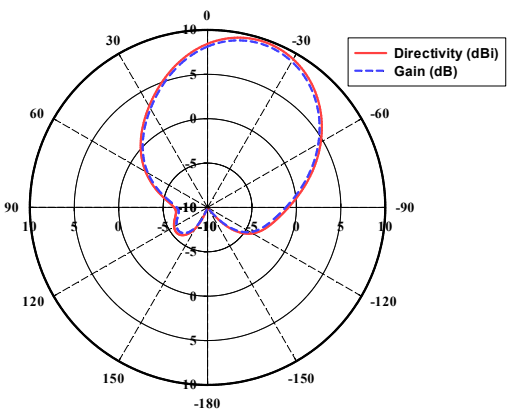

(c)

Figure 6. Second case of E-plane: (a) Diode1 is ON state and diode2 OFF state, (b) Radiation pattern is directed to the direction of $-20^{\circ}$ and (c) Power patterns in the E-plane at theta $=-20^{\circ}$

\subsection{Radiation pattern control on azimuth $(Z)$ plane}

For controlling radiation pattern in the Z-plane, both of diode1 and diode2 has been switched ON. The switching will be either in the diode 3 or diode 4, First case when the diode on the upper side (diode3) has been switched OFF and at the same time the diode at the down side of antenna (diode4) have been switched ON as shown in Figure 7(a), the stop band will appear on the down side of antenna and the pass band will present at the upper side of antenna. The simulated power pattern of the antenna with EBG is directed at the Z-plane of the microstrip patch antenna from the down side of antenna (stop band when connecting vias) to the opposite upper side of antenna (pass band when disconnecting vias) in the direction of $+20^{\circ}$ as shown in Figure 7(b). The simulation results of EBG illustrate its capability to control the power pattern directivity around the Z-plane of the microstrip patch antenna. By alternately switching diode the EBG sector surface characteristic will change also.

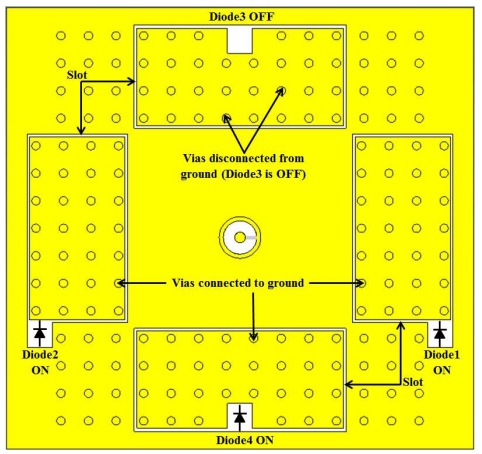

(a)

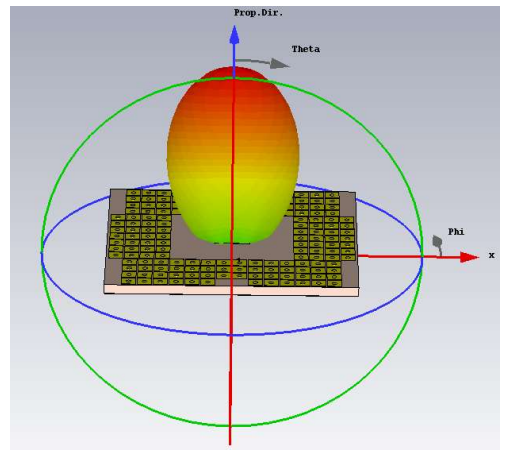

(b)

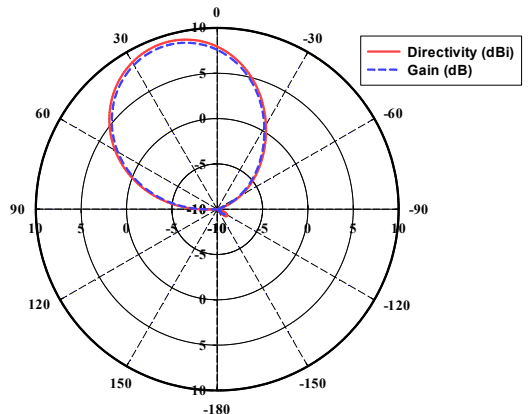

(c)

Figure 7. First Case of Z-plane: (a) Diodes (1,2and4) are setting as ON state and Diode3 is OFF state, (b) Radiation Pattern is directed to the direction of $+18^{\circ}$ and (c) Power patterns in the Z-plane at theta $=+18^{\circ}$ 
For this case, to direct the pattern towards the upper sector, pin vias at the sector have been disconnected from the ground plane (diode3 switched OFF), whereas pin vias have been connected to ground plane of antenna for the down sector through switched the diode4 at ON state. The surface wave has propagated towards upper sector (pass band) and reflected from the down sector (stop band). Figure 7(c) shows the Z-plane power pattern, which is directed towards the upper sector at $\mathrm{phi}=+18^{\circ}$ with the maximum gain and directivity which equal to $8.8 \mathrm{~dB}$ and $9.2 \mathrm{dBi}$ respectively.

Second case for controlling the radiation pattern in Z-plane, the direction will be from the upper side of antenna (stop band ) where the diode 3 is ON state to the down side of antenna (pass band) where the diode4 is OFF state as shown in Figure 8(a). The simulated power pattern of the antenna with EBG is directed from the upper side of antenna (stop band ) where the pin vias has been connected to the ground plane(diode3 ON) to the down side of antenna (pass band) where the pin vias has been disconnected from the ground plane(diode4 ON) in the direction of $-18^{\circ}$ as shown in Figure $8(\mathrm{~b})$. The placing of mushroom EBGs unit cell around the antenna clarifies its capability to control the radiation pattern directivity around the Z-plane. By alternately switching diode, the EBG sector surface characteristic will change also. To control the beam direction, pin vias have been connected and disconnected at each sector. For example, to direct the radiation pattern towards the down sector, pin vias at the sector have been disconnected from the ground plane (diode4 switched OFF), whereas pin vias have been connected to ground plane of antenna for the upper sector through connecting the slot on the ground plane by switched the diode3at ON state. The surface wave has propagated towards down sector (pass band) and reflected from the upper sector (stop band). Figure 8(c) shows the Z-plane radiation pattern, which is directed towards the down sector at phi $=-18^{\circ}$ with the maximum gain of $8.6 \mathrm{~dB}$ and directivity $9.1 \mathrm{dBi}$.

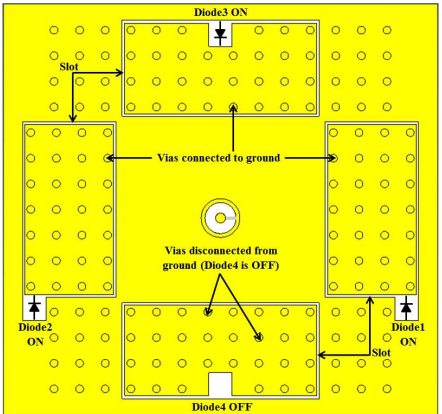

(a)

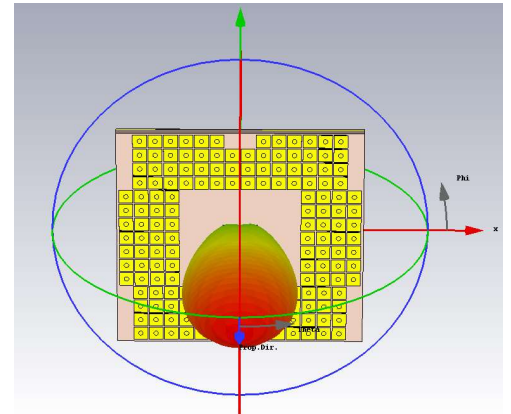

(b)

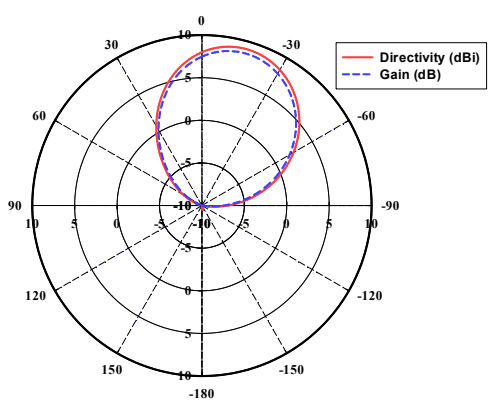

(c)

Figure 8. Second Case of Z-plane: (a) Diodes (1,2and4) are setting as ON state and Diode3 is OFF state, (b) Radiation Pattern is directed to the direction of $-18^{\circ}$ and (c) Power patterns in the Z-plane at theta $=-18^{\circ}$

The impact of surface wave in the antenna radiation pattern is considered unwanted,where it increase the side lobe level and decrease the efficiency of antenna. By using high dielectric constant we achieved compact size of antenna, but the side effect for doing this, is the anarrow band width. In order to avoid the narrow band width due to the high dielectric constant, we will increas the substrate thickness. The surface waves will also increases when increasing the thickness of substrate, this problem can be solved by using EBG with connecting pin vias between the ground and the patch of antenna (short circuit) to suppress the surface waves in the same band of frequency (i.e. 6GHz). The efficiency enhancement of antenna for all cases when using the EBG for surrounding the rectangular patch antenna as shown in Figure 9. Reference Antenna (without EBG) has efficiency equal to $80 \%$ and after applying the EBG with the same antenna, the results for all cases have been stated in the Table 1 . When all of the diodes have been switched $\mathrm{ON}$ state (short circuit) we achieved the better improvement in $0^{\circ}$ main lobe direction, the efficiency becomes $96.5 \%$. For the case of E-plane radiation pattern control, the changing were either in diode1 or diode2. For example when the diode2 (left side of antenna) remaining on the ON state (short circuit) and the changing was on the diode1 (right side of antenna) to OFF state (open circuit), the efficiency improved by $95 \%$ in $+20^{\circ}$ main lobe direction has been achieved. Next the diode1 on the ON state (short circuit) and the changing will be on the diode 2 to OFF state (open circuit), the same improvement has been achieved in efficiency by $95 \%$ but in $-20^{\circ}$ main lobe direction. For the case of Z-plane radiation pattern control, switching diodes were related to the diode 3 and diode4 in the same situation of the previous case (E-plane radiation pattern control). The efficiency improved by $92 \%$ in $+18^{\circ}$ main lobe direction when the 
diode 3 is OFF state (upper side of antenna) and when the diode4 has been switched OFF (down side of antenna) the efficiency improved by $90 \%$ in $-18^{\circ}$ main lobe direction.

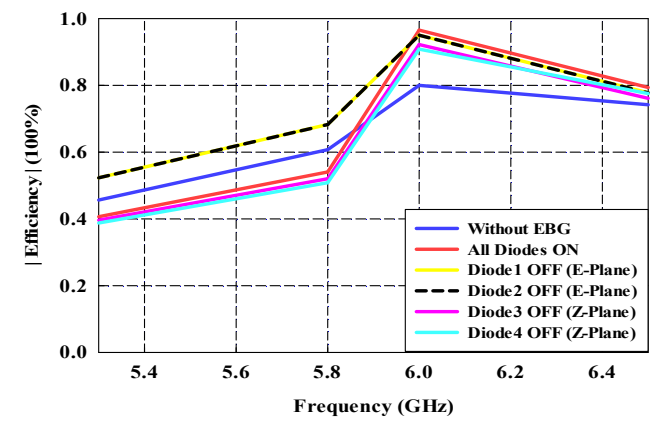

Figure 9. Efficiency improvement by using EBG

Table 1. Switchable EBG Results on Antenna's Radiation Pattern Control

\begin{tabular}{cccccccc}
\hline Diode 1 & Diode 2 & Diode 3 & Diode 4 & Direction & Gain $(\mathrm{dB})$ & Directivity $(\mathrm{dBi})$ & Efficiency $(\%)$ \\
\hline ON & ON & ON & ON & $0^{\circ}$ & 9.86 & 10 & 96.5 \\
OFF & ON & ON & ON & $+20^{\circ}$ (E-Plane) & 9.4 & 9.7 & 95 \\
ON & OFF & ON & ON & $-20^{\circ}$ (E-Plane) & 9.4 & 9.7 & 95 \\
ON & ON & OFF & ON & $+18^{\circ}$ (Z-Plane) & 8.8 & 9.2 & 92 \\
ON & ON & ON & OFF & $-18^{\circ}$ (Z-Plane) & 8.6 & 9.1 & 90 \\
\hline
\end{tabular}

The results of the reference antenna (without using switchable EBG) for the gain, directivity and efficiency were $4.74 \mathrm{~dB}, 5.66 \mathrm{dBi}$ and $80 \%$ respectively. From the above result in Table 1 , a good improvement after using EBG has been achieved in both of elevation and azimuth planes. The results in E-plane were better than it in Z-plane, because of the surface waves appears in E-plane more than it in Z-plane [28]. As known the EBG working on surface waves, in case of increasing surface waves the bandgap feature of EBG will give better result due to high performance for suppressing surface waves and in the same time we insured the EBG design has been done in correct way.

\section{CONCLUSION}

In this paper a novel antenna structure is designed by combining the mushroom-like EBG structure for suppressing surface waves with the switchable diodes to control radiation pattern of microstrip patch antenna in elevation and azimuth planes. Unconventional radiation pattern control is a unique characteristic of this design; the main lobe gain is approximately constant for all steering angles in E-plane with unnoticeable degradation of gain in Z-plane direction. EBG of square cells are bounded the patch antenna and arranged in symmetrical way for the two opposite sides, By surrounding the rectangular patch antenna with 4 columns for all the sides, only the columns in the left and right sides are shifted inwards to antenna edges by $0.3 \mathrm{~mm}\left(0.06 \lambda_{0}\right)$. At the ground plane of antenna the diodes have been switched ON and OFF states, the EBG sector properties in stop band (connecting vias) and pass band (disconnecting vias) are altered, when the diode switched OFF in specific sector the radiation pattern will directing into that sector. The simulation results show the flexibility in the radiation pattern control for the both azimuth $\left(-18^{\circ}<\varphi<18^{\circ}\right)$ and elevation planes $\left(-20^{\circ}<\theta<20^{\circ}\right)$ by using minimal number of diodes (four diodes). Best improvement in main lobe direction when all of the diodes have been switched $\mathrm{ON}$, directivity of $10 \mathrm{dBi}$, gain $9.86 \mathrm{~dB}$ and efficiency $96.5 \%$ at the operating frequency of $6 \mathrm{GHz}$. The results in E-plane angles direction were $9.4 \mathrm{~dB}$, $9.7 \mathrm{~dB}$ and $95 \%$ for the gain, directivity and efficiency respectively, while the E-plane angles direction results were $8.8 \mathrm{~dB}, 9.2 \mathrm{~dB}$ and $92 \%$ for the gain, directivity and efficiency respectively. This structure has great possible for the wireless and radar applicatio.

\section{ACKNOWLEDGEMENTS}

This work was supported by UTeM Zamalah Scheme. The authors would also like to thank Center for Research and Innovation Management (CRIM), Centre of Excellence, UTeM, UTeM's research grant JURNAL/2018/FKEKK/J00001 and Universiti Teknikal Malaysia Melaka (UTeM) for their encouragement and help in sponsoring this study. 


\section{REFERENCES}

[1] M. Tarbouch, A. El Amri, and H. Terchoune, "Design, Realization and Measurements of Compact Dual-band, " Int. J. Electr. Comput. Eng., vol. 8, no. 1, pp. 172-178, 2018.

[2] S. M. Shah et al., "Frequency Reconfiguration Mechanism of a PIN Diode on a Reconfigurable Antenna for LTE and WLAN Applications, " Int. J. Electr. Comput. Eng., vol. 8, no. 3, pp. 1893-1901, 2018.

[3] D. Sievenpiper, L. Zhang, R. F. Jimenez Broas, N. G. Alexöpolous, and E. Yablonovitch, "High-impedance Electromagnetic Surfaces with a Forbidden Frequency Band, "IEEE Trans. Microw. Theory Tech., vol. 47, no. 11, pp. 2059-2074, 1999.

[4] F. R. Yang, K. P. Ma, M. Yongxi Qian, and T. Itoh, "A uniplanar compact photonic-bandgap (uc-pbg) structure and its applications for microwave circuits, " IEEE Trans. Microw. Theory Tech., vol. 47, no. 8, pp. 1509-1514, 1999.

[5] S. Yamini and B. Panjavarnam, "Microstrip Patch Antenna Integrated with EBG, " Proc. 2017 2nd Int. Conf. Comput. Commun. Technol. ICCCT 2017, pp. 41-45, 2017.

[6] R. Gonzalo, P. De Maagt, and M. Sorolla, "Enhanced Patch-Antenna Performance by Suppressing Surface Waves Using Photonic-Bandgap Substrates," IEEE Trans. Microw. Theory Tech., vol. 47, no. 11, pp. 2131-2138, 1999.

[7] J. S. Colburn, "Patch Antennas on Externally Perforated High Dielectric Constant Substrates, " IEEE Trans. Antennas Propag., vol. 47, no. 12, pp. 1785-1794, 1999.

[8] A. M. S. Diaz, M. C. Galaz-Larios, and E. Ramirez-Garcia, "Analysis and Design of Electromagnetic Band Gap Structures with Application In Planar Antennas, " 2017 42nd Int. Conf. Infrared, Millimeter, Terahertz Waves (IRMMW-THz).Cancun, Mex., pp. 1-2, 2017.

[9] S. Chen, X. Zhao, Y. Yao, J. Ma, and S. Yang, "Study on Radiation Performances of Dipole Antenna with Diamond-Structure EBG Substrate Fabricated by 3D Printing Technique, " Int. J. Appl. Ceram. Technol., pp. 1-5, Jan 2018

[10] A. Altaf, M. A. Alsunaidi, and E. Arvas, "A Novel EBG Structure to Improve Isolation in MIMO Antenna, " IEEE, pp. 105-106, 2017.

[11] C. Neo and Y. H. Lee, "Patch Antenna Enhancement using a Mushroom-like EBG Structures, " IEEE Antennas Propag. Soc. AP-S Int. Symp., vol. 1, pp. 614-615, 2013.

[12] M. K. Mohsen et al., "The Fundamental of Leaky Wave Antenna, " J. Telecommun. Electron. Comput. Eng., vol. 10, no. 1, pp. 119-127, 2018.

[13] M. K. Mohsen, M. S. M. Isa, Z.Zakaria, A. A. M. Isa, M. K. Abdulhameed, and M. L. Attiah, "Control Radiation Pattern for Half Width Microstrip Leaky Wave Antenna by Using PIN Diodes, " Int. J. Electr. Comput. Eng., vol. 8, no. 5, 2018.

[14] M. K. Mohsen, M. Saari, M. Isa, and M.K.Abdulhameed, "Novel Design and Implementation of MIMO Antenna for LTE Application, " J. Telecommun. Electron. Comput. Eng., vol. Vol. 10, no. July, pp. 43-49, 2018.

[15] W. Ebg and V. Reflector, "Gain Improvement of MSAs Array by V sing Curved, " Int. Electr. Eng. Congr. IEEE, pp. 4-7, 2014.

[16] J. Zaid, M. Farahani, A. Kesavan, and T. A. Denidni, "Miniaturized Microstrip Patch Antenna Using Electromagnetic Band Gap ( EBG ) structures for Multiport Passive UHF RFID-Tag Applications, " IEEE, pp. 2459-2460, 2017.

[17] M. K. Abdulhameed, M. S. M. Isa, Z.Zakaria, I.M.Ibrahim, M. K. Mohsen, and M. L. Attiah, "Controlling The Radiation Pattern of Patch Antenna Using Switchable EBG," Telecomminication Computing Electronics and Control (TELKOMNIKA), vol. 16, no. 5, 2018.

[18] J. Costantine, Y. Tawk, S. E. Barbin, and C. G. Christodoulou, "Reconfigurable antennas: Design and applications," Proc. IEEE, vol. 103, no. 3, pp. 424-437, 2015.

[19] C. G. Christodoulou, Y. Tawk, S. A. Lane, and S. R. Erwin, "Reconfigurable Antennas for Wireless and Space Applications, " Proc. IEEE, vol. 100, no. 7, pp. 2250-2261, 2012.

[20] M. S. Mohamad Isa, R. J. Langle, and S. Khamas, "Antenna Pattern Diversity Using EBG, " 2010 Loughbrgh. Antennas Propag. Conf. LAPC 2010, no. November, pp. 325-328, 2010.

[21] M. S. M. Isa et al., "Antenna Beam Steering using Sectorized Square EBG, " J. Telecommun. Electron. Comput. Eng., vol. 4, no. 1, pp. 39-44, 2012.

[22] M. J. Al-Hasan, T. A. Denidni, and A. R. Sebak, "Millimeter-wave EBG-based antenna pattern diversity, " IEEE Antennas Propag. Soc. AP-S Int. Symp., pp. 272-273, 2013.

[23] C. Huang, C. Ji, X. Wu, J. Song, and X. Luo, "Combining FSS and EBG Surfaces for High-Efficiency Transmission and Low-Scattering Properties," IEEE Trans. Antennas Propag., vol. 66, no. 3, pp. 1628-1632, 2018.

[24] Q. R. Zheng, Y. Q. Fu, and N. C. Yuan, "A Novel Compact Spiral Electromagnetic Band-Gap (EBG) Structure," IEEE Trans. Antennas Propag., vol. 56, no. 6, pp. 1656-1660, 2008.

[25] M. K. Abdulhameed, M. S. M. Isa, I. M. Ibrahim, and M. K. Mohsin, "Improvement of Microstrip Antenna Performance on Thick and High Permittivity Substrate with Electromagnetic Band Gap," Jour Adv Res. Dyn. Control Syst., vol. 10, no. 4, pp. 661-669, 2018.

[26] M. K. Abdulhameed, M. S. M. Isa, Z.Zakaria, M. K.Mohsin, and M. L. Attiah, "Mushroom-Like EBG to Improve Patch Antenna Performance For C-Band Satellite Application, " Int. J. Electr. Comput. Eng., vol. 8, no. 5, 2018.

[27] S. Jain and S. Dwivedi, "Design and Optimization of H-Shaped Multi-Band Microstrip Patch Antenna," Int. J. Eng. Sci. Manag. Res., vol. 3, pp. 22-26, 2016.

[28] F. Yang and Y. Rahmat-Samii, "Microstrip Antennas Integrated with Electromagnetic Band-Gap (EBG) Structures: A Low Mutual Coupling Design for Array Applications," IEEE Trans. Antennas Propag., vol. 51, no. 10 II, pp. 2936-2946, 2003. 